\title{
Dengue or Japanese encephalitis?
}

\author{
Pallavi Sehgal $^{1}$, Wasil Rasool Sheikh ${ }^{2}$, Dheeraj Nair ${ }^{1}$, Ankur Verma $^{2}$, Sanjay Jaiswal ${ }^{2}$ \\ ${ }^{1}$ Department of Emergency Medicine, Max Super Superspeciality Hospital, Vaishali, UP, India; ${ }^{2}$ Department of Emergency \\ Medicine, Max Super Speciality Hospital, Patparganj, New Delhi-110092, India.
}

\section{Corresponding Author:}

Dr. Pallavi Sehgal

Email: pallavisehga115@gmail.com

This is an Open Access article distributed under the terms of the Creative Commons Attribution License (creativecommons.org/ licenses/by/3.0).

Received

Accepted

Published

May 15, 2017

August 22, 2017

August 30, 2017

\begin{abstract}
Background: Dengue and Japanese encephalitis are considered as one of the most common arbovirus diseases in tropics. Dengue virus has a wide spectrum of presentation starting from the classical dengue fever to dengue encephalitis and dengue shock syndrome. Moreover, Japanese encephalitis, which is one of the major public health problems in most of the South East Asian countries including India, may present with similar clinical picture mimicking dengue encephalitis. Case Report: We report the case of a 34 year old male patient who presented to the emergency department with complaints of fever and subsequently developed altered mental status. Investigations of the patient revealed dengue NS1 antigen positive but Magnetic Resonance Imaging findings were suggestive of Japanese encephalitis. Conclusion: Here, we discuss the likelihood of DengueJapanese encephalitis co-infection in endemic countries where both dengue and Japanese encephalitis are common.
\end{abstract}

Keywords: Arboviruses, Dengue Virus, Fever, India, Japanese Encephalitis.

\section{Introduction}

Dengue and Japanese encephalitis are considered as one of the most common arboviral diseases in tropics. Dengue viral infection presents in very unpredictable manner varying from silent infection to uncomplicated dengue fever, dengue hemorrhagic fever and fulminant dengue shock syndrome. Various neurological manifestations in dengue fever and dengue hemorrhagic fever have always been thought as secondary to vasculitis with extravasation of fluid, cerebral hypo-perfusion, edema, hypo-natremia, hepatic or renal failure, termed as dengue encephalopathy [1]. Japanese encephalitis, which is one of the major public health problems in most of the South East Asian countries including India, may present with similar clinical pictures mimicking dengue encephalitis.

\section{Case Report}

A 34 year old male was brought to the Emergency Department (ED) with complaints of high grade

(C) 2017 Journal of Case Reports fever associated with chills and rigors since three days. Patient also complained of having multiple episodes of vomiting with streaks of blood. There was no history of bleeding from any other site. On presentation patient was conscious and oriented and had a heart rate of $120 / \mathrm{min}$, blood pressure of $110 / 70 \mathrm{~mm} \mathrm{Hg}$, respiratory rate of $20 / \mathrm{min}$, temperature of $100^{\circ} \mathrm{F}$ and oxygen saturation of $100 \%$ on room air. Rest of the systemic examination was unremarkable. During his stay in the ED the patient developed episodes of projectile vomiting, and went into a state of altered mentation. Repeat vital signs showed severe hypotension for which the patient received intravenous fluids and inotropes. In view of deteriorating mental status with an inability to protect his airway, the patient was electively intubated and attached to mechanical ventilator. Arterial blood gas analysis revealed severe metabolic acidosis. Blood investigation revealed a platelet count of $30 \times 10^{9} / \mathrm{L}$ and dengue virus NS1 antigen test was positive. Magnetic Resonance Imaging (MRI) brain was done which 
revealed extensive bilateral symmetrical areas of relative restriction of diffusion and hyper-intense signal alteration on T2W and FLAIR in cerebral white matter, thalamus, brainstem and cerebellum; mass effect over lateral, $3^{\text {rd }}$ and fourth ventricle with mild tonsillar herniation, suggestive of Japanese encephalitis [Fig.1].

A diagnosis of dengue fever with Japanese encephalitis was made and patient was admitted in Intensive Care Unit (ICU) under the neurology team. Treatment was continued with inotropic support, anti-pyretics, platelet transfusions and supportive care over the next few days but patient's condition continued to deteriorate and he expired on the $4^{\text {th }}$ day.

\section{Discussion}

Dengue virus is a single stranded RNA virus belonging to the Flaviridae genus, and is classified into 4 serotypes [1-4]. Serotypes 2 and 3 are mostly responsible for all the neurological manifestations [4,5]. Even though, the complete mechanism through which dengue virus crosses blood brain barrier is not clear, it is proposed that the virus enters the blood brain barrier through infected macrophages [6]. Severe dengue infection can manifest with neurological complications. Management of dengue is supportive and vaccinations for prevention are under trials.

Japanese encephalitis (JE) virus is classically a neurotropic virus. It is responsible for most of the cases of viral encephalitis in many countries of Asia accounting for 68,000 clinical cases every year. JE virus doesn't give rise to any systemic manifestations but has great affinity for midbrain, striatum, thalamus and anterior horn cells of spinal cord. JE doesn't have any specific antiviral therapy and the management is mainly symptomatic, however it can be prevented by vaccination. Solomon et al. reported case series of 9 cases with dengue encephalitis [DE], out of which all patients were positive for dengue

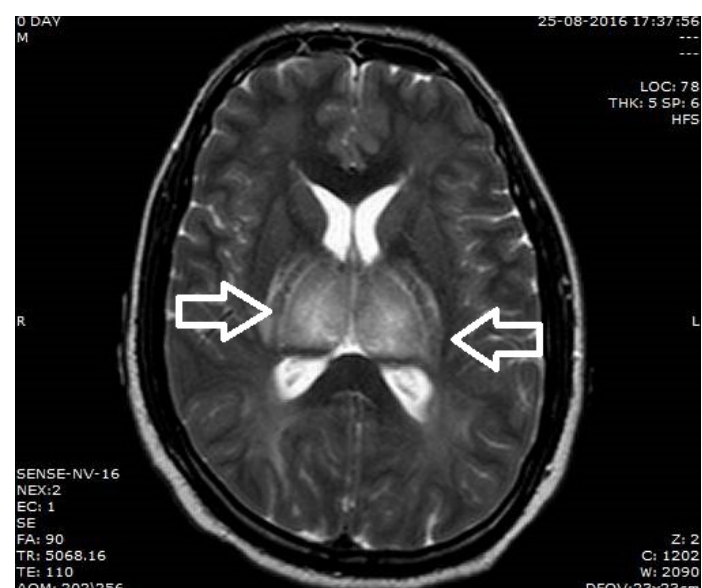

Fig.1: Extensive bilateral symmetrical areas of relative restriction of diffusion and hyper-intense signal alteration on $T 2 W$ and FLAIR in cerebral white matter, thalamus, brainstem and cerebellum, mass effect over lateral, $3^{\text {rd }}$ and fourth ventricle with mild tonsillar herniation.

serology but virus/antibodies for dengue were seen only in cerebrospinal fluid of two patients. In their case series, seven patients didn't have typical manifestations of DE [4].

Borawake et al. reported a case of DE that had MRI findings suggestive of lesions in bilateral thalamus, pons and cerebellar regions [7]. These findings are more sensitive in cases of Japanese encephalitis [8]. A study has been done which established a relationship between pre-existing JE virus antibodies and severity of subsequent dengue virus infection. It was noted that antibodies for JE virus were associated with increased prevalence of symptomatic dengue infection [9]. A recent study noted that patients who were admitted for dengue virus infection, also turned out to be seropositive for JE [10].

The case that we are reporting presented with fever and subsequently developed altered mental status and shock. Above stated MRI findings are seen in more than half of patients suffering from Japanese encephalitis [11]. In this case, the patient had atypical symptoms with dengue serology positive and MRI findings suggestive of features of JE. 


\section{Conclusion}

There is a possibility of dual infection or cross reactivity between $\mathrm{JE}$ and dengue which needs to be considered, if clinical and serological evidences are seen in the same patient. In a country known for dengue epidemics, it is important for emergency physicians to keep a differential of JE in dengue positive patients with altered mental status.

Contributors: PS: manuscript writing, literature search; WRS, DN: manuscript editing, literature search; AV, SJ: Study designing and manuscript editing. PS will act as guarantor. All authors approved the final version of manuscript. Funding: None; Competing interests: None stated.

\section{References}

1. Cam BV, Fonsmark L, Hue NB, Phuong NT, Poulsen A, Heegaard ED. Prospective case-control study of encephalopathy in children with dengue haemorrhagic fever. Am J Trop Med Hyg. 2001;65:848-851.

2. Hendarto SK, Hadinegoro SR. Dengue encephalopathy. Acta Pediatr Jpn. 1992;34:350-357.

3. Nathanson N, Cole GA. Immunosuppression and experimental virus infection of the nervous system. Adv Virus Res. 1970;16:397-428.
4. Solomon T, Dung NM, Vaughn DW, Kneen R, Thao LT, Raengsakulrach B, et al. Neurological manifestations of dengue infection. Lancet. 2000;355:1053-1059.

5. Carod-Artal FJ, Wichmann O, Farrar J, Gascón J. Neurological complications of dengue virus infection. Lancet Neurol. 2013;12:906-919.

6. Misra UK, Kalita J, Syam UK, Dhole TN. Neurological manifestations of dengue virus infection. J Neurol Sci. 2006;244:117-122.

7. Borawake K, Prayag P, Wagh A, Dole S. Dengue encephalitis. Indian J Crit Care Med. 2011;15:190-193.

8. Kumar S, Misra UK, Kalita J, Salwani V, Gupta RK, Gujral R. MRI in Japanese Encephalitis. Neuroradiology. 1997;39:180-184.

9. Anderson KB, Gibbons RV, Thomas SJ, Rothman AL, Nisalak A, Berkelman RL, et al. Preexisting Japanese encephalitis virus neutralizing antibodies and increased symptomatic dengue illness in a school-based cohort in Thailand. PLoS Negl Trop Dis. 2011;5:e1311.

10. Jeewandara C, Gomes L, Paranavitane SA, Tantirimudalige M, Panapitiya SS, Jayewardene A, et al. Change in dengue and Japanese encephalitis sero-prevalence rates in Sri Lanka. PLoS One. 2015;10:e0144799.

11. Shohji H, Murakami T, Murai I, Kida H, Sato Y, Kojima $\mathrm{K}$, et al. A follow up study by CT and MRI in 3 cases of Japanese encephalitis. Neuroradiology. 1990;32:215219. 